\title{
HGMN STARS AS APPARENT X-RAY EMITTERS
}

\section{S. HUBRIG}

University of Potsdam, Am Neuen Palais 10, D-14469 Potsdam, Germany

AND

T. W. BERGHÖFER

Space Sciences Laboratory, University of California, Berkeley, CA 94720-7450, USA

\section{Introduction}

In the ROSAT all-sky survey $11 \mathrm{HgMn}$ stars were detected as soft X-ray emitters (Berghöfer, Schmitt \& Cassinelli 1996). Prior to ROSAT, X-ray observations with the Einstein Observatory had suggested that stars in the spectral range B5-A7 are devoid of X-ray emission. Since there is no X-ray emitting mechanism available for these stars (also not for HgMn stars), the usual argument in the case of an X-ray detected star of this spectral type is the existence of an unseen low-mass companion which is responsible for the X-ray emission. However, this hypothesis is not easily testable. Based on high resolution X-ray images taken with the ROSAT HRI, Berghöfer \& Schmitt (1994) showed that known visual late-type companions can be disregarded in this context. In almost all cases studied so far (including two HgMn stars in our sample) the X-ray emission is associated with the primary B star.

The purpose of the present work is to use all available data for our sample of X-ray detected HgMn stars and conclude on the nature of possible spectroscopic companions. We emphasize that some of our sample stars consist of two nearly equal B stars. The observed X-ray emission in these systems is also inconsistent with the secondary star and, thus, a third component must exist to explain the X-ray emission by a low-mass companion. Some of our sample stars show X-ray luminosities that exceed the $\mathrm{X}$-ray output of normal late-type stars and, therefore, an active pre-main sequence companion (PMS) is required. This hypothesis is supported by 
the fact that a significant fraction of the HgMn stars found in the ROSAT survey belong to rather young stellar groups like the Pleiades supercluster or the Sco-Cen association.

Here we describe our method to conclude on the nature of possible lowmass companions. In a first step stellar masses and ages were derived for our sample of HgMn stars. For this we utilized the stellar model grids provided by Schaller et al. (1992); the stellar distances were taken from the recently released Hipparcos catalog and the effective temperatures were compiled from the literature. We then assumed that the absence of a secondary in the optical spectrum implies a mass ration of $\mathrm{M}_{1} / \mathrm{M}_{2} \geq 1.5$ for the two binary components and all systems are formed coeval. A further criterion was the saturation limit of $\log \left(\mathrm{L}_{x} / \mathrm{L}_{B o l}\right) \approx-3$ known for late-type star $\mathrm{X}$-ray emission (cf. Schmitt 1997); for the observed X-ray luminosities this relation provides upper limits for the bolometric luminosities of the possible secondaries. Together with these limits for the companions masses, luminosities, and ages, we utilized the pre-main-sequence evolutionary tracks provided by D'Antona and Mazzitelli (1994) to limit the range of possible companions of the $11 \mathrm{HgMn}$ stars.

\section{Results and Conclusions}

For all of our sample HgMn stars detected in the ROSAT all-sky survey we find that a late-type companion can provide a natural explanation for the observed X-ray emission. In 7 cases (HD 32964, HD 33904, HD 35497, HD 75333, HD 110073, HD 141556, and HD 173524) the detected X-ray emission can be explained by a main-sequence late-type star, whereas for the stars HD 27295, HD 27376, HD 29589, and HD 221507 a PMS star is required. Further investigations by means of radial velocity studies and high-resolution imaging (e.g., in the near IR) are needed to detect the predicted companions. According to the mass lower limits derived for possible companions in our sample of $\mathrm{HgMn}$ stars spectral types are in the range late K-M4. It is remarkable that in many cases when a spectroscopic binary with a late-B primary has a third, distant companion, the SB primary is a HgMn star (e.g., Hubrig \& Mathys 1995).

\section{References}

Berghöfer, T.W., Schmitt, J.H.M.M.: 1994, A\&A 292, L5

Berghöfer, T.W., Schmitt, J.H.M.M., Cassinelli, J.P.: 1996, A\&AS 118, 481

D'Antona F., Mazzitelli I.: 1994, ApJ Suppl. 90, 467

Hubrig S., Mathys G.: 1995, Comm. Astroph. 18, 167

Schaller G., Schaerer D., Meynet G., Maeder A.: 1992, A\&AS 96, 269

Schmitt, J.H.M.M.: 1997, A\&A 318, 215 